\title{
Mortalidade por todas as causas e por doenças cardiovasculares em três estados do Brasil, 1980 a 2006
}

\author{
Gabriel Porto Soares, ${ }^{1}$ Júlia Dias Brum, ${ }^{1}$ \\ Gláucia Maria Moraes de Oliveira, ${ }^{1}$ Carlos Henrique Klein ${ }^{2}$ \\ e Nelson Albuquerque de Souza e Silva ${ }^{1}$
}

Como citar Soares GP, Brum JD, Oliveira GMM, Klein CH, Souza e Silva NA. Mortalidade por todas as causas e por doenças cardiovasculares em três estados do Brasil, 1980 a 2006. Rev Panam Salud Publica. 2010;28(4):258-66.

RESUMO Objetivo. Avaliar a mortalidade por todas as causas e por doenças do aparelho circulatório (DAC), doenças isquêmicas do coração (DIC) e doenças cerebrovasculares (DCBV) de 1980 a 2006 no Rio de Janeiro, Rio Grande do Sul, São Paulo e em suas capitais, considerando o impacto dos óbitos por causas mal definidas.

Métodos. Os dados de população e óbitos foram obtidos no Banco de Dados do Sistema Único de Saúde. As taxas de mortalidade para as doenças de interesse e para as causas mal definidas foram ajustadas pelo método direto para adultos acima de 20 anos. Como as taxas por causas mal definidas no Rio de Janeiro aumentaram muito a partir de 1990, foram estimados percentuais de mortalidade proporcional por causas mal definidas. Empregaram-se modelos de regressão linear para análise das tendências.

Resultados. Observou-se uma queda relevante da mortalidade por todas as causas nos três estados e capitais. O Rio de Janeiro e sua capital apresentaram taxas mais elevadas. A mortalidade por DAC apresentou queda mais acentuada do que a mortalidade por todas as causas. A mortalidade proporcional por causas mal definidas no Estado do Rio de Janeiro e sua capital superou aquela das demais localidades a partir de 1990. A mortalidade por DCBV apresentou declinio, mais acentuado no Estado do Rio de Janeiro e sua capital. O Estado do Rio de Janeiro apresentou também taxas de mortalidade mais elevadas por DIC até 1993. Entre as capitais, São Paulo apresentou a partir de 1992 as taxas mais elevadas de mortalidade por DIC.

Conclusão. A queda da mortalidade por todas as causas se deveu principalmente ao declínio da mortalidade por DAC. A queda da mortalidade por DAC se deveu em parte à redução da mortalidade por DCBV, notadamente no Estado do Rio de Janeiro.

Palavras-chave Mortalidade; causas de morte; doenças cardiovasculares; isquemia miocárdica; transtornos cerebrovasculares; epidemiologia; Brasil.

1 Universidade Federal do Rio de Janeiro, Programa de Pós-Graduação em Medicina (Cardiologia). Correspondência: gp.soares@yahoo.com.br (Gabriel Porto Soares)

2 Escola Nacional de Saúde Pública (ENSP), Fundação Oswaldo Cruz, Rio de Janeiro (RJ), Brasil.
Na Europa, as taxas de mortalidade por todas as causas têm apresentado redução desde o século XVIII. Na Inglaterra, no século XIX, ocorreu queda de três quartos na taxa de mortalidade geral
(1). Da mesma forma, a partir do final da década de 1950, iniciou-se nos países industrializados um declínio da mortalidade por doenças do aparelho circulatório (DAC), as principais causas de morte 
tanto nos países desenvolvidos quanto nos países em desenvolvimento. Esse declínio foi observado principalmente nos Estados Unidos, Canadá, Austrália e países do Oeste da Europa (1).

O Brasil também apresentou queda da mortalidade por todas as causas; contudo, a grande redução só ocorreu nos últimos 60 anos, com queda superior a 60\% (2), de 18/1000 em 1940 (1) para 5,5/1 000 em 2006 (3). As DAC, por sua vez, representavam um terço de todos os óbitos e quase $30 \%$ do total de mortes na faixa etária de 20 a 59 anos de idade no ano de 2006, atingindo a população adulta em plena fase produtiva (4). Dentre as DAC, destacaram-se as doenças cerebrovasculares (DCBV) e as doenças isquêmicas do coração (DIC). Em 2006, essas duas doenças representaram, respectivamente, 32 e $30 \%$ dos óbitos por DAC no Brasil (3).

Considerando localidades específicas, uma tendência de queda nas taxas de mortalidade por DAC foi constatada no Estado e Município de São Paulo $(5,6)$ e nos municípios de Salvador (7), Goiânia (8) e Porto Alegre (9). Atribuiu-se essa redução ao melhor controle dos fatores de risco cardiovascular, especialmente da hipertensão arterial sistêmica, às novas técnicas diagnósticas, aos avanços no campo terapêutico e à melhoria das condições socioeconômicas (1, 10, 11). Mansur et al. observaram quedas na mortalidade por DAC, DIC e DCBV na maioria das capitais de 11 estados brasileiros no período de 1979 a 1996 (12). Rio de Janeiro e Porto Alegre apresentaram uma redução no risco de morte por DIC e DCBV. Ainda no período de 1979 a 1996, São Paulo apresentou um discreto aumento nas DIC na faixa etária de 30 a 39 anos em ambos os sexos e nas mulheres de 40 a 59 anos. Por outro lado, os mesmos autores (12) mencionam altas taxas de mortalidade por causas mal definidas nas regiões Nordeste, Norte e CentroOeste, mas não consideram o impacto da mortalidade crescente por causas mal definidas no Rio de Janeiro a partir de 1990, nem a influência que essas taxas podem ter sobre as tendências observadas para as taxas de mortalidade de causas definidas, como as DAC (11).

O objetivo do presente estudo foi avaliar e comparar a mortalidade de adultos de 20 anos ou mais por todas as causas, DAC, DIC e DCBV no período de 1980 a 2006 nos estados do Rio de Janeiro, Rio
Grande do Sul e São Paulo e em suas capitais, levando em consideração o impacto dos óbitos por causas mal definidas nos dados sobre mortalidade.

\section{MATERIAIS E MÉTODOS}

Este é um estudo descritivo de séries históricas de 1980 a 2006 de mortalidade por todas as causas, DAC, DIC, DCBV e causas mal definidas. Foram analisados dados de adultos com 20 anos ou mais dos Estados do Rio de Janeiro (RJest), Rio Grande do Sul (RSest) e São Paulo (SPest); e de suas capitais, respectivamente Rio de Janeiro (RJcap), Porto Alegre (RScap) e São Paulo (SPcap). Os dados referentes às populações de 1980 a 2006 foram obtidos do Banco de Dados do Sistema Único de Saúde (DATASUS) (3). A escolha dessas três unidades da federação e de suas capitais baseou-se na boa qualidade de preenchimento dos atestados de óbito nesses locais, inferida pelas taxas menores de mortalidade proporcional por causas mal definidas e pelo maior número de afecções registradas por atestado $(11,13)$.

Os dados referentes aos óbitos por todas as causas, DAC, DIC, DCBV e causas mal definidas também foram obtidos no site do DATASUS. Foram pesquisadas as causas básicas de óbito segundo a Nona Revisão da Classificação Internacional de Doenças de 1975 (CID-9) (14) para os óbitos de 1980 a 1995: capítulo VII para DAC, códigos 410 a 414 para DIC, códigos 430 a 438 para DCBV e capítulo XVI para CMD. Para os óbitos de 1996 a 2006, foi utilizada a Décima Revisão da Classificação Internacional de Doenças e Causas de Morte (CID-10) (15), com o capítulo IX para DAC, códigos de I20 a I25 para DIC, códigos de I60 a I69 para DCBV e capítulo XVIII para CMD.

Calcularam-se taxas de mortalidade por todas as causas, DAC, DIC e DCBV e causas mal definidas para adultos de 20 anos ou mais. Foram calculadas as taxas brutas e ajustadas, por sexo e idade, pelo método direto $(16,17)$, para os estados e suas capitais. A população padrão para os ajustamentos foi a aferida pelo Censo de 2000 no Estado do Rio de Janeiro, estratificada em sete grupos etários (20 a 29 anos; 30 a 39 anos; 40 a 49 anos; 50 a 59 anos; 60 a 69 anos; 70 a 79 anos; e 80 anos ou mais). Como as taxas de mortalidade por causas mal definidas no Estado do
Rio de Janeiro aumentaram de forma relevante a partir de 1990 (18), optou-se pela realização de compensação, que consistiu em alocar para os óbitos por DAC, DIC e DCBV parte dos óbitos por causas mal definidas. Assim, a proporção de óbitos mal definidos foi ajustada para refletir a proporção correspondente de óbitos definidos em relação a todos os óbitos, excluídos os mal definidos. Após a compensação dos óbitos por DAC, DIC e DCBV pelos de causas mal definidas, foram estimadas novas taxas de mortalidade, ajustadas por sexo e idade. Essas novas taxas foram denominadas de compensadas e ajustadas. As taxas de mortalidade por outras causas de óbito que não DAC foram obtidas pela diferença entre todas as causas e DAC, com o objetivo de demonstrar a relação entre a queda da mortalidade por todas as causas e a redução da mortalidade por DAC. A compensação foi utilizada nos três estados e suas capitais. Foram estimados percentuais de mortalidade proporcional (16) por causas mal definidas em todos os anos estudados.

Foram construídos gráficos das taxas de mortalidade por todas as causas, DAC, DIC e DCBV para os anos de 1980 a 2006. Nesses gráficos foi empregado o método de médias móveis (19) de 3 anos para suavizar o efeito de flutuações anuais. Para determinado ano, a mortalidade é a média aritmética do ano anterior, do representado e do seguinte. Empregaram-se modelos de regressão linear para a análise das tendências das taxas de mortalidade compensadas e ajustadas por DIC e DCBV, que foram consideradas variáveis dependentes Y. Os anos foram considerados como variáveis independentes X. Esses modelos serviram para estimar as médias das taxas de mortalidade, diferenças anuais médias e percentuais de incrementos anuais no período estudado. Para o ajustamento de taxas e demais manobras quantitativas foi utilizado o programa STATA.

\section{RESULTADOS}

As tabelas 1, 2 e 3 apresentam, respectivamente, as taxas brutas de mortalidade por DAC, DCBV e DIC. Todos os estados e municípios apresentaram queda nas taxas de mortalidade por essas causas, com redução mais acentuada na mortalidade por DCBV do que por DIC. As seis áreas estudadas apre- 
TABELA 1. Taxas brutas de mortalidade por 100000 habitantes por doenças do aparelho circulatório nos adultos de 20 anos ou mais, Rio de Janeiro, Rio Grande do Sul e São Paulo, 1980 a 2006

\begin{tabular}{|c|c|c|c|c|c|c|}
\hline Ano & RJcap ${ }^{a}$ & RJest ${ }^{a}$ & $\mathrm{RScap}^{\mathrm{a}}$ & $\mathrm{RSest}^{\mathrm{a}}$ & SPcap $^{a}$ & SPest $^{\mathrm{a}}$ \\
\hline 1980 & 530,4 & 511,1 & 409,4 & 455,3 & 381,0 & 429,7 \\
\hline 1981 & 507,0 & 506,9 & 418,0 & 433,7 & 365,6 & 415,7 \\
\hline 1982 & 500,8 & 486,6 & 399,7 & 417,3 & 356,4 & 398,4 \\
\hline 1983 & 525,3 & 509,3 & 412,3 & 436,6 & 367,2 & 406,1 \\
\hline 1984 & 524,6 & 510,3 & 418,1 & 441,7 & 357,2 & 403,1 \\
\hline 1985 & 536,4 & 525,1 & 405,4 & 418,3 & 364,0 & 398,5 \\
\hline 1986 & 545,0 & 520,9 & 407,6 & 407,8 & 358,3 & 386,6 \\
\hline 1987 & 545,7 & 520,7 & 414,5 & 418,3 & 357,9 & 380,5 \\
\hline 1988 & 560,2 & 534,5 & 457,0 & 440,5 & 380,5 & 400,6 \\
\hline 1989 & 545,0 & 518,1 & 386,6 & 404,9 & 365,0 & 388,3 \\
\hline 1990 & 521,2 & 500,9 & 413,8 & 404,9 & 359,0 & 383,8 \\
\hline 1991 & 497,3 & 472,6 & 380,5 & 386,8 & 335,8 & 353,9 \\
\hline 1992 & 477,5 & 456,5 & 385,9 & 393,1 & 338,3 & 354,7 \\
\hline 1993 & 500,1 & 485,6 & 388,9 & 406,2 & 350,2 & 370,7 \\
\hline 1994 & 484,5 & 471,2 & 374,6 & 395,2 & 347,9 & 370,5 \\
\hline 1995 & 461,6 & 459,2 & 376,7 & 402,2 & 343,5 & 360,5 \\
\hline 1996 & 474,2 & 451,3 & 378,7 & 372,0 & 365,6 & 355,2 \\
\hline 1997 & 454,3 & 427,9 & 364,6 & 349,9 & 356,8 & 345,5 \\
\hline 1998 & 462,4 & 432,4 & 398,7 & 379,7 & 351,6 & 339,8 \\
\hline 1999 & 435,7 & 417,6 & 384,4 & 364,8 & 359,4 & 340,4 \\
\hline 2000 & 371,4 & 362,8 & 352,0 & 356,3 & 316,9 & 317,2 \\
\hline 2001 & 395,9 & 384,5 & 352,3 & 347,3 & 317,8 & 317,2 \\
\hline 2002 & 408,8 & 391,9 & 352,6 & 346,1 & 313,9 & 311,2 \\
\hline 2003 & 384,4 & 377,9 & 347,0 & 341,1 & 310,8 & 312,0 \\
\hline 2004 & 386,1 & 382,0 & 312,6 & 344,6 & 318,2 & 318,0 \\
\hline 2005 & 365,8 & 357,5 & 322,3 & 324,3 & 294,7 & 296,0 \\
\hline 2006 & 381,2 & 372,2 & 335,5 & 323,3 & 301,7 & 304,3 \\
\hline
\end{tabular}

a RJcap = capital do Rio de Janeiro; RJest = Estado do Rio de Janeiro; RScap = capital do Rio Grande do Sul; RSest = Estado do Rio Grande do Sul; SPcap = capital de São Paulo; SPest = Estado de São Paulo.

TABELA 2. Taxas brutas de mortalidade por 100000 habitantes por doenças cerebrovasculares em adultos de 20 anos ou mais, Rio de Janeiro, Rio Grande do Sul e São Paulo, 1980 a 2006

\begin{tabular}{|c|c|c|c|c|c|c|}
\hline Ano & RJcap ${ }^{a}$ & RJest $^{\mathrm{a}}$ & RScap ${ }^{a}$ & RSest $^{\mathrm{a}}$ & SPcap ${ }^{a}$ & SPest $^{a}$ \\
\hline 1980 & 216,3 & 210,5 & 122,2 & 160,7 & 127,7 & 151,3 \\
\hline 1981 & 187,6 & 197,0 & 127,8 & 157,2 & 123,7 & 147,0 \\
\hline 1982 & 198,8 & 192,8 & 122,6 & 153,5 & 116,8 & 139,0 \\
\hline 1983 & 195,7 & 198,4 & 125,1 & 158,3 & 118,1 & 139,7 \\
\hline 1984 & 196,9 & 201,8 & 126,5 & 158,8 & 116,7 & 141,4 \\
\hline 1985 & 194,7 & 202,8 & 122,7 & 149,6 & 116,9 & 138,2 \\
\hline 1986 & 193,2 & 197,9 & 120,2 & 147,7 & 112,8 & 131,4 \\
\hline 1987 & 190,9 & 194,6 & 128,2 & 149,3 & 107,6 & 126,0 \\
\hline 1988 & 187,7 & 192,3 & 131,6 & 150,9 & 115,5 & 130,6 \\
\hline 1989 & 180,0 & 183,0 & 137,6 & 147,8 & 108,2 & 126,1 \\
\hline 1990 & 180,1 & 184,9 & 135,5 & 148,2 & 107,6 & 123,1 \\
\hline 1991 & 170,9 & 175,4 & 118,5 & 141,3 & 97,7 & 110,2 \\
\hline 1992 & 174,7 & 178,2 & 120,6 & 142,6 & 97,5 & 111,4 \\
\hline 1993 & 180,6 & 186,3 & 129,5 & 147,8 & 98,1 & 115,8 \\
\hline 1994 & 172,4 & 178,2 & 110,1 & 140,0 & 98,4 & 114,7 \\
\hline 1995 & 160,9 & 169,2 & 118,9 & 139,9 & 96,5 & 109,6 \\
\hline 1996 & 154,1 & 158,8 & 115,2 & 120,9 & 102,7 & 108,4 \\
\hline 1997 & 153,0 & 151,0 & 111,6 & 118,5 & 101,2 & 105,8 \\
\hline 1998 & 151,6 & 150,3 & 127,1 & 127,7 & 98,2 & 102,8 \\
\hline 1999 & 142,4 & 142,1 & 112,2 & 118,9 & 100,6 & 103,5 \\
\hline 2000 & 130,5 & 132,1 & 105,7 & 117,0 & 86,0 & 95,5 \\
\hline 2001 & 129,1 & 127,9 & 121,6 & 116,5 & 82,7 & 92,7 \\
\hline 2002 & 130,9 & 128,0 & 114,8 & 115,5 & 83,8 & 91,4 \\
\hline 2003 & 123,8 & 124,8 & 120,2 & 117,7 & 79,9 & 88,5 \\
\hline 2004 & 121,5 & 120,6 & 108,5 & 117,5 & 82,5 & 90,1 \\
\hline 2005 & 109,8 & 110,7 & 118,1 & 112,3 & 76,0 & 82,6 \\
\hline 2006 & 115,2 & 115,3 & 116,6 & 109,0 & 77,9 & 85,8 \\
\hline
\end{tabular}

a RJcap = capital do Rio de Janeiro; RJest = Estado do Rio de Janeiro; RScap = capital do Rio Grande do Sul; RSest = Estado do Rio Grande do Sul; SPcap = capital de São Paulo; SPest = Estado de São Paulo. sentaram variações semelhantes em suas taxas durante o período.

A tabela 4 apresenta as taxas de mortalidade brutas e padronizadas, as diferenças e os incrementos percentuais da mortalidade por todas as causas, DAC e outras causas (OutC) nos estados e capitais estudados. Observamos que todos apresentaram queda nas taxas ajustadas de mortalidade por todas as causas, com redução ainda mais acentuada das taxas de DAC. Em termos absolutos, de acordo com as taxas brutas, houve aumento, mas isso se deveu a modificações no perfil etário das populações, que envelheceram. Portanto, considerando-se uma população de perfil etário e sexual estável (a do Estado do Rio de Janeiro em 2000), houve queda percentual em todas as populações. Ao comparar tanto capitais como estados, verificamos que as taxas de mortalidade mais elevadas foram sempre as do Rio de Janeiro. Já Rio Grande do Sul e São Paulo alternaram sua posição no decorrer do período em relação às mortalidades totais e cardiovasculares: as taxas brutas do Rio Grande do Sul foram mais elevadas do que as de São Paulo, enquanto que as padronizadas foram mais elevadas em São Paulo do que no Rio Grande do Sul. Só não houve troca de posição entre os dois estados para a mortalidade bruta e padronizada por causas não cardiovasculares.

$\mathrm{Na}$ figura 1A observa-se que ocorreu queda relevante da mortalidade por todas as causas do início para o fim do período nos três estados e capitais. Podese observar, grosso modo, quatro fases distintas. A primeira fase é marcada por relativa estabilidade das taxas de mortalidade por todas as causas até 1991. A segunda mostra elevação até 1994. A terceira mostra queda significativa até 2001 e a quarta mostra um retorno à estabilidade, em patamar inferior aos anos iniciais. O Estado do Rio de Janeiro e sua capital apresentaram as taxas mais elevadas em todos os anos. Nos estados do Rio Grande do Sul e São Paulo e em suas capitais o comportamento das curvas foi semelhante, com períodos de sobreposição das mesmas.

Na figura 1B a mortalidade por DAC apresenta, nos três estados e capitais, padrão descendente em todo o período, com queda mais acentuada quando comparada à mortalidade por todas as causas. A mortalidade por DAC no Estado do Rio de Janeiro foi superior durante todo o pe- 
TABELA 3. Taxas brutas de mortalidade por 100000 por doenças isquêmicas do coração em adultos de 20 anos ou mais, Rio de Janeiro, Rio Grande do Sul e São Paulo, 1980 a 2006

\begin{tabular}{|c|c|c|c|c|c|c|}
\hline Ano & RJcap ${ }^{a}$ & RJest $^{\mathrm{a}}$ & RScap ${ }^{a}$ & RSest $^{\mathrm{a}}$ & SPcap ${ }^{a}$ & SPest ${ }^{a}$ \\
\hline 1980 & 186,9 & 166,2 & 161,9 & 148,4 & 140,4 & 142,0 \\
\hline 1981 & 183,8 & 165,6 & 179,7 & 151,5 & 135,7 & 138,6 \\
\hline 1982 & 176,0 & 157,4 & 164,3 & 142,0 & 131,5 & 132,1 \\
\hline 1983 & 193,4 & 169,8 & 168,0 & 148,0 & 137,9 & 137,8 \\
\hline 1984 & 194,1 & 172,5 & 184,3 & 155,5 & 135,5 & 134,9 \\
\hline 1985 & 197,3 & 176,3 & 173,9 & 150,1 & 137,6 & 132,6 \\
\hline 1986 & 203,5 & 176,9 & 174,1 & 140,9 & 133,6 & 129,7 \\
\hline 1987 & 205,4 & 175,7 & 179,7 & 149,4 & 137,2 & 130,1 \\
\hline 1988 & 208,8 & 179,8 & 194,6 & 156,4 & 140,5 & 134,1 \\
\hline 1989 & 207,1 & 178,6 & 140,2 & 134,1 & 136,8 & 130,2 \\
\hline 1990 & 190,4 & 164,7 & 159,8 & 138,0 & 135,5 & 129,7 \\
\hline 1991 & 177,3 & 150,7 & 156,7 & 135,5 & 129,3 & 122,0 \\
\hline 1992 & 159,0 & 137,0 & 157,1 & 136,7 & 129,0 & 118,9 \\
\hline 1993 & 165,5 & 143,7 & 143,1 & 137,2 & 132,8 & 121,4 \\
\hline 1994 & 163,4 & 140,4 & 151,4 & 139,1 & 131,5 & 122,4 \\
\hline 1995 & 154,3 & 140,0 & 147,3 & 139,3 & 131,3 & 120,5 \\
\hline 1996 & 168,1 & 142,9 & 144,3 & 134,8 & 144,2 & 120,3 \\
\hline 1997 & 157,4 & 134,5 & 140,9 & 128,4 & 140,3 & 117,6 \\
\hline 1998 & 165,5 & 138,0 & 165,5 & 139,8 & 137,4 & 115,6 \\
\hline 1999 & 152,9 & 133,8 & 158,5 & 138,1 & 140,8 & 116,6 \\
\hline 2000 & 134,7 & 122,1 & 152,9 & 139,1 & 133,6 & 114,8 \\
\hline 2001 & 137,5 & 124,1 & 141,1 & 128,7 & 128,4 & 110,9 \\
\hline 2002 & 143,5 & 126,9 & 144,6 & 126,0 & 128,6 & 110,3 \\
\hline 2003 & 130,8 & 118,4 & 129,8 & 120,5 & 127,5 & 111,4 \\
\hline 2004 & 132,8 & 120,6 & 121,8 & 123,5 & 126,3 & 111,8 \\
\hline 2005 & 130,0 & 113,9 & 117,1 & 112,6 & 112,5 & 101,4 \\
\hline 2006 & 132,3 & 117,3 & 129,0 & 114,9 & 116,7 & 103,7 \\
\hline
\end{tabular}

a RJcap = capital do Rio de Janeiro; RJest = Estado do Rio de Janeiro; RScap = capital do Rio Grande do Sul; RSest = Estado do Rio Grande do Sul; SPcap = capital de São Paulo; SPest = Estado de São Paulo.

TABELA 4. Taxas de mortalidade médias brutas e taxas compensadas e ajustadas por todas as causas, por doenças do aparelho circulatório e por demais causas, diferenças anuais médias e percentual de incrementos anuais médios estimados em adultos de 20 anos ou mais, Rio de Janeiro, Rio Grande do Sul e São Paulo, 1980 a 2006

\begin{tabular}{|c|c|c|c|c|c|c|c|}
\hline \multirow[b]{2}{*}{ Local } & \multirow[b]{2}{*}{ Causa } & \multicolumn{3}{|c|}{ Brutas } & \multicolumn{3}{|c|}{ Taxas compensadas e ajustadas ${ }^{b}$} \\
\hline & & Média & $\begin{array}{c}\text { Diferença } \\
\text { anual }\end{array}$ & $\% \operatorname{lnc} c$ & Média & $\begin{array}{c}\text { Diferença } \\
\text { anual }\end{array}$ & $\% \operatorname{lnc}^{c}$ \\
\hline \multicolumn{8}{|c|}{ Rio de Janeiro } \\
\hline \multirow[t]{3}{*}{ Capital } & Todas & 1175,1 & 2,4 & 0,2 & 1192,0 & $-9,3$ & $-0,8$ \\
\hline & $\mathrm{DAC}^{\mathrm{d}}$ & 473,4 & $-7,0$ & $-1,5$ & 487,3 & $-13,0$ & $-2,7$ \\
\hline & Demais & 701,6 & 9,3 & 1,3 & 704,7 & 3,7 & 0,5 \\
\hline \multirow[t]{3}{*}{ Estado } & Todas & 1115,3 & 0,7 & 0,1 & 1228,0 & $-9,2$ & $-0,8$ \\
\hline & $\mathrm{DAC}^{\mathrm{d}}$ & 457,3 & $-6,7$ & $-1,5$ & 521,7 & $-12,9$ & $-2,5$ \\
\hline & Demais & 658,0 & 7,4 & 1,1 & 706,3 & 3,7 & 0,5 \\
\hline \multicolumn{8}{|c|}{ Rio Grande do Sul } \\
\hline \multirow{3}{*}{ Capital } & Todas & 1011,6 & 5,1 & 0,5 & 1095,5 & $-7,5$ & $-0,7$ \\
\hline & $\mathrm{DAC}^{\mathrm{d}}$ & 383,3 & $-3,5$ & $-0,9$ & 423,7 & $-10,0$ & $-2,4$ \\
\hline & Demais & 628,3 & 8,6 & 1,4 & 671,7 & 2,5 & 0,4 \\
\hline \multirow[t]{3}{*}{ Estado } & Todas & 965,1 & 2,6 & 0,3 & 1069,4 & $-6,9$ & $-0,6$ \\
\hline & $D^{d} C^{d}$ & 389,3 & $-4,6$ & $-1,2$ & 447,4 & $-10,1$ & $-2,3$ \\
\hline & Demais & 575,8 & 7,2 & 1,2 & 622,0 & 3,2 & 0,5 \\
\hline \multicolumn{8}{|l|}{ São Paulo } \\
\hline \multirow[t]{3}{*}{ Capital } & Todas & 901,2 & 2,9 & 0,3 & 1104,2 & $-7,1$ & $-0,6$ \\
\hline & $D^{\prime d}$ & 345,7 & $-2,5$ & $-0,7$ & 451,4 & $-8,8$ & $-2,0$ \\
\hline & Demais & 555,4 & 5,3 & 1,0 & 652,8 & 1,7 & 0,3 \\
\hline \multirow[t]{3}{*}{ Estado } & Todas & 921,0 & 0,3 & 0,0 & 1118,6 & $-8,1$ & $-0,7$ \\
\hline & $D^{\prime d}$ & 361,4 & $-4,7$ & $-1,3$ & 467,6 & $-10,5$ & $-2,3$ \\
\hline & Demais & 559,6 & 5,0 & 0,9 & 651,0 & 2,5 & 0,4 \\
\hline
\end{tabular}

a Estimativas por regressão linear; dados por 100000 habitantes.

b Compensação pelas causas mal definidas e ajustamento por idade e sexo pelo método direto, tendo como população padrão o Estado do Rio de Janeiro em 2000.

c Percentual de incremento anual médio.

d Doenças do aparelho circulatório. ríodo, ao passo que sua capital se aproximou dos demais estados e capitais a partir de 1994. Porto Alegre apresentou as menores taxas em todos os anos.

A figura 1C apresenta a mortalidade por outras causas, excluídas as doenças do aparelho circulatório. Foi observada elevação da mortalidade por outras causas nos três estados e capitais até 1994; a partir de então houve queda até o início dos anos 2000. As taxas se elevaram no Estado de São Paulo e em sua capital até 2006, enquanto os estados e capitais de Rio de Janeiro e Rio Grande do Sul mantiveram estabilidade em suas taxas nesse final do período.

As figuras 2A e 2B apresentam a mortalidade proporcional por causas mal definidas nos estados e capitais, respectivamente. O Estado de São Paulo apresentou mortalidades proporcionais constantes, em torno de 6\%, até 1995. Depois disso houve queda para cerca de $4 \%$, com retorno ao nível anterior a partir do ano 2000. O Estado do Rio Grande do Sul declinou de $10 \%$ no início do período para $2 \%$ em 1996, voltando a subir a partir de 2000 até um pouco acima de 5\%. O Estado do Rio de Janeiro, que possuía taxas de mortalidade proporcional mais baixas durante a década iniciada em 1980, passou a superar os demais estados a partir do ano de 1991, tendo atingido quase $12 \%$ em 2000, apresentando ainda taxa superior a 9\% em 2006. Porto Alegre e a capital São Paulo apresentaram, durante todo o período, taxas razoavelmente constantes, quase sempre abaixo de $2 \%$. Já a capital Rio de Janeiro, que durante a década iniciada em 1980 quase sempre apresentou taxas proporcionais por causas mal definidas pouco acima de $2 \%$, passou por um incremento notável durante toda a década iniciada em 1990, até atingir cerca de $11 \%$ em 2000 . O pico de causas mal definidas observado em Porto Alegre em 1989 pode ter sido influenciado pelo fato, noticiado à época na imprensa local, de que um médico vendeu mais de 1500 atestados de óbito cujas causas tiveram de ser consideradas como mal definidas, conforme informado por A. Fischmann, da Secretaria Municipal de Saúde de Porto Alegre (comunicação pessoal, 2007).

As figuras $3 \mathrm{~A}$ e $3 \mathrm{~B}$ descrevem as taxas de mortalidade por DCBV nos estados e capitais, respectivamente. Todas as curvas apresentaram declínio. O declínio mais acentuado ocorreu no Rio de Ja- 
FIGURA 1. Mortalidade por todas as causas ajustada (A), por doenças do aparelho circulatório (B) e por outras causas (C) compensadas e ajustadas em adultos de 20 anos ou mais, Rio de Janeiro, Rio Grande do Sul e São Paulo, 1980 a 2006
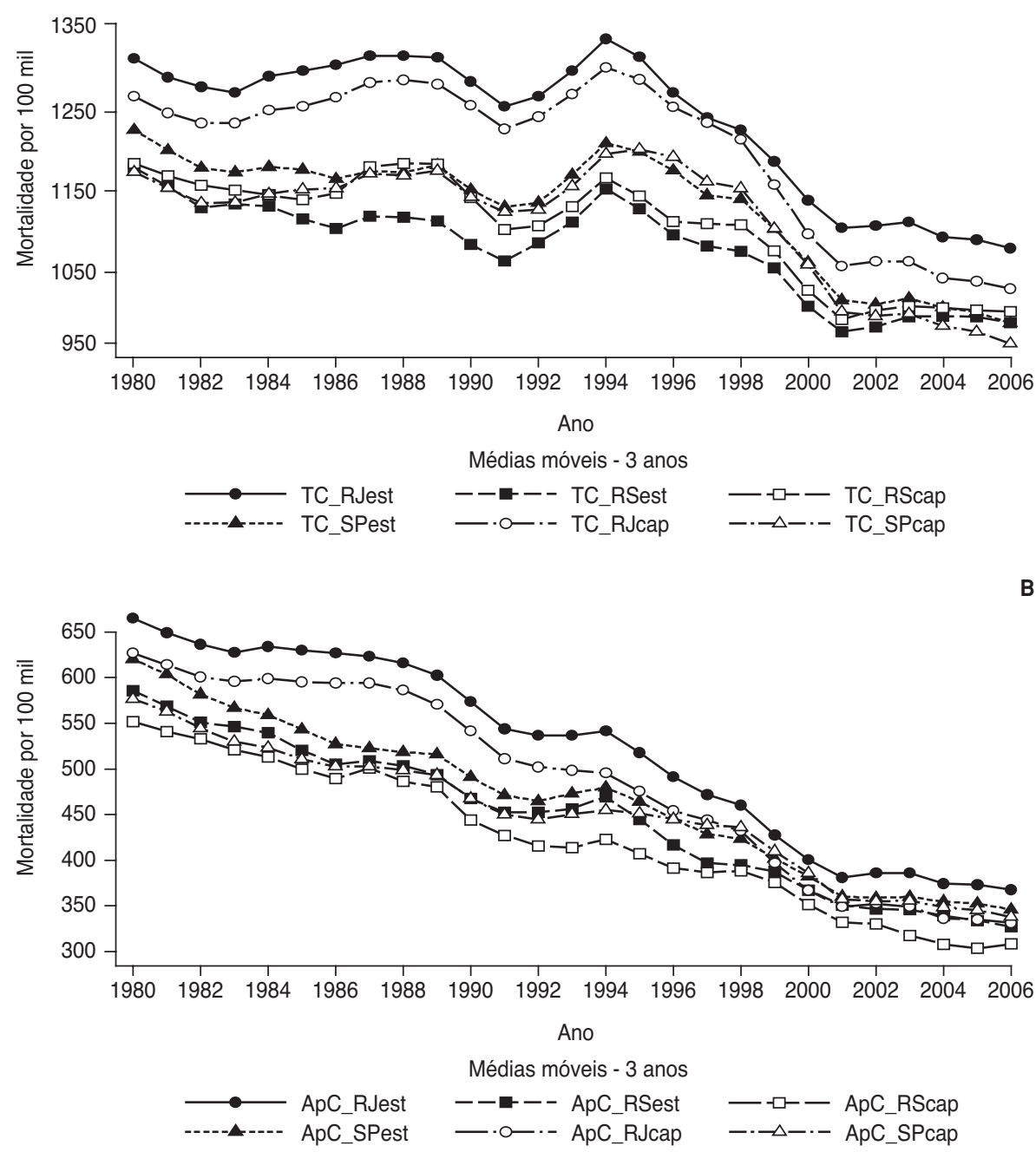

C

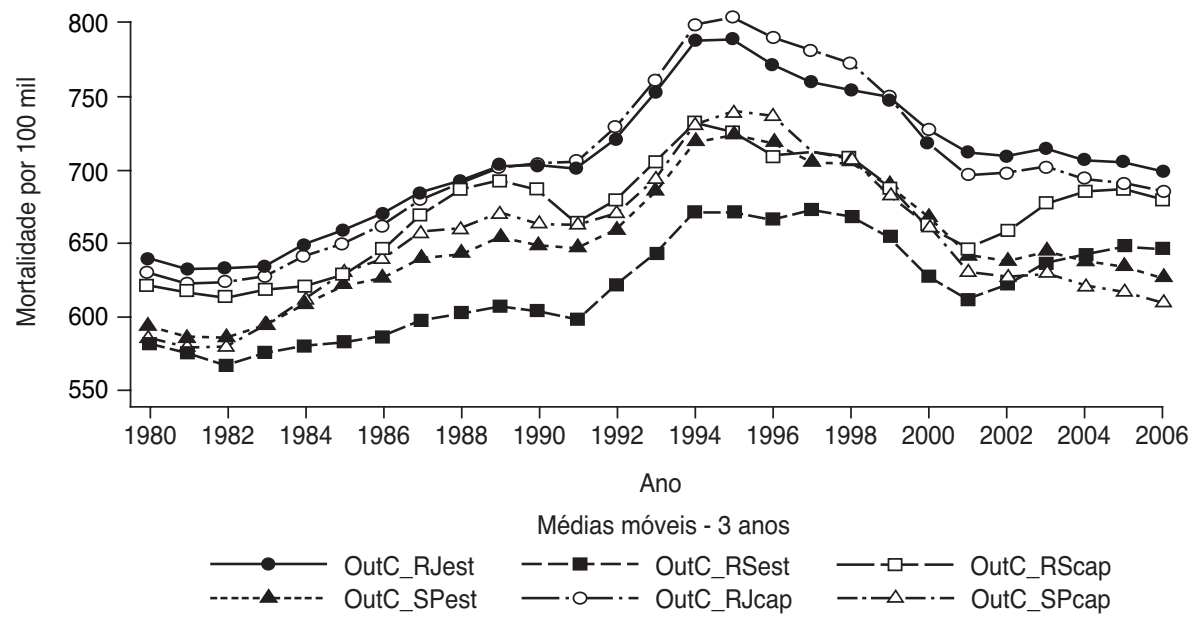

a RJcap = capital do Rio de Janeiro; RJest = Estado do Rio de Janeiro; RScap = capital do Rio Grande do Sul; RSest = Estado do Rio Grande do Sul; SPcap = capital de São Paulo; SPest = Estado de São Paulo. neiro e em sua capital, que passaram a se confundir com os demais estados no final do período.

As taxas de mortalidade por DIC nos estados e capitais estão descritas nas figuras $4 \mathrm{~A}$ e $4 \mathrm{~B}$, respectivamente. O Estado do Rio de Janeiro apresentou as taxas de mortalidade mais elevadas por DIC até 1993. A partir daí, as taxas são semelhantes para os três estados. As capitais de Rio de Janeiro e Rio Grande do Sul apresentaram taxas semelhantes durante todo o período, sendo superiores à taxa para a capital São Paulo até o início da década de 1990. A partir de 1992, a capital São Paulo passou a apresentar taxas de mortalidade por DIC constantemente mais elevadas, até 2006.

\section{DISCUSSÃO}

O presente estudo revelou uma queda relevante da mortalidade por todas as causas em todos os estados e capitais analisados. No Brasil, um decréscimo da mortalidade por todas as causas foi observado nos últimos 60 anos do século $X X$, atribuído principalmente a medidas de saúde pública e técnicas de medicina preventiva. Segundo Prata (1) e Yunes e Ronchezel (2), a taxa de mortalidade geral reflete contribuições de medidas de saúde somadas ao nível de desenvolvimento econômico, agindo principalmente na redução da mortalidade infantil e no aumento da expectativa de vida.

No Brasil, no decorrer do século XX, houve uma importante mudança no perfil epidemiológico da mortalidade por grupos de causas específicas. Prata, em estudo do período de 1930 a 1985, observou que, do início do século até o fim da década de 1960, as doenças infecciosas eram a primeira causa de óbito. Depois disso, passou a predominar a mortalidade por DAC, que permanece até hoje como principal causa de óbito no Brasil. Também observou-se um gradativo incremento na mortalidade por neoplasias e causas externas, que atualmente se alternam como segunda causa de óbito, dependendo da região avaliada (1).

As informações compiladas dos registros de óbito somente passaram a ser confiáveis no Brasil a partir do final da década de 1970, sendo que o Ministério da Saúde do Brasil passou a publicar os dados sobre mortalidade somente em 1979. Assim, foram publicados diversos estudos sobre a mortalidade por DAC 
FIGURA 2. Mortalidade proporcional por causas mal definidas (\%) nos estados (A) e capitais (B) do Rio de Janeiro, Rio Grande do Sul e São Paulo em adultos de 20 anos ou mais, 1980 a 2006
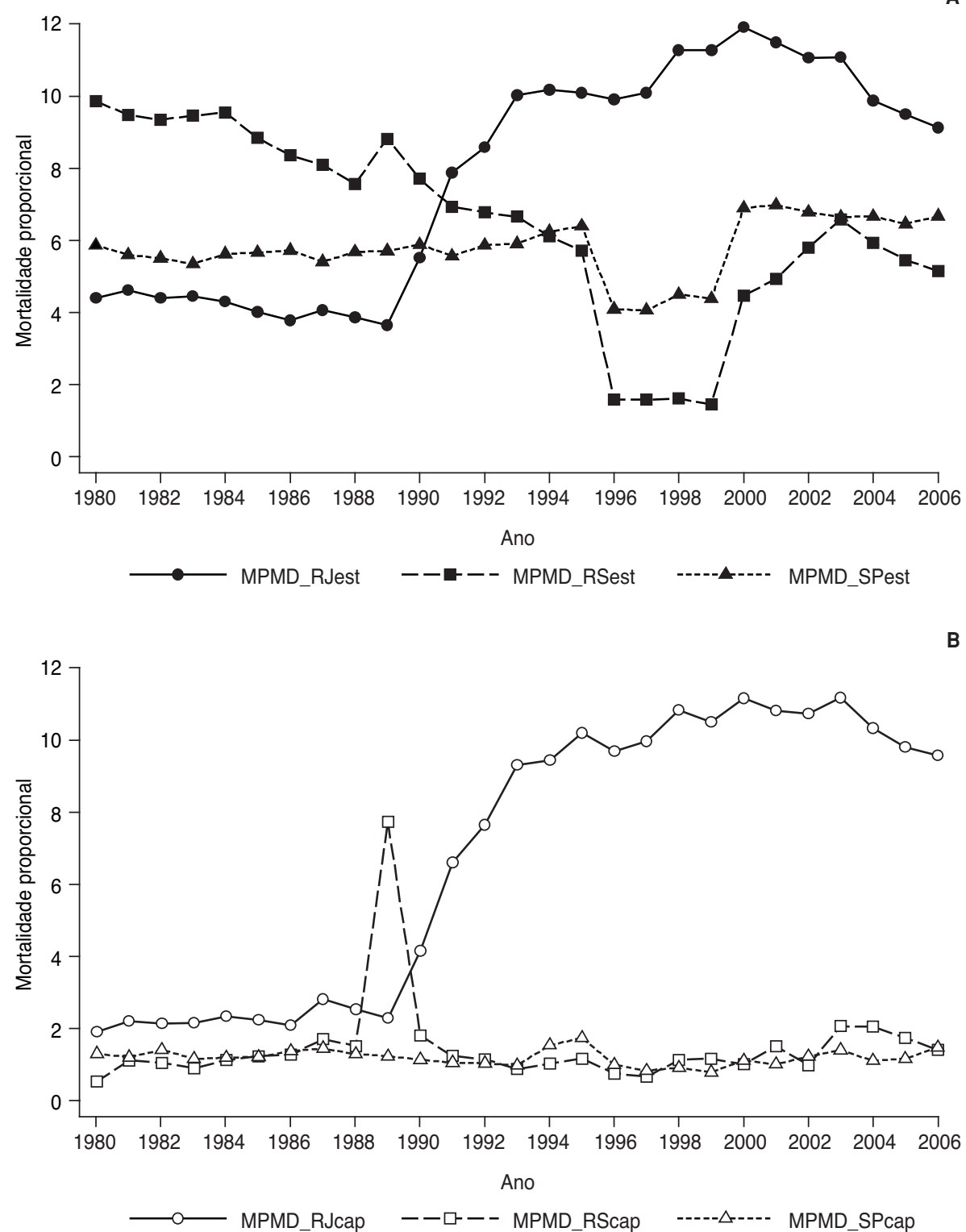

${ }^{\text {a }}$ RJcap = capital do Rio de Janeiro; RJest = Estado do Rio de Janeiro; RScap = capital do Rio Grande do Sul; RSest = Estado do Rio Grande do Sul; SPcap = capital de São Paulo; SPest = Estado de São Paulo.

com dados gerados a partir da década de $1970(1,5-8,11,12,20)$. Laurenti (21), em 1981, sinalizou o aumento nas DIC, que representavam as principais causas básicas de morte $(16,7 \%)$ em adultos de 15 a 74 anos no Município de São Paulo. Em outro estudo, esse mesmo autor chamou a atenção para a preponderância das DCBV sobre as DIC em várias capitais brasileiras, e para o fato de que esse quadro se assemelha ao observado no Japão, onde também ocorre tal predominância (22).
Chor et al. (23) compararam as taxas de mortalidade no Brasil, Estados Unidos, Inglaterra e Cuba em 1988, e constataram que cerca de $50 \%$ dos óbitos masculinos por DIC ocorriam antes dos 65 anos de idade no Brasil, contra 25\% nos outros países. Os autores ponderam que a população brasileira tem perfil mais jovem do que a população dos demais países incluídos na comparação. O mesmo ocorreu em relação ao risco de morte por DCBV, que foi maior no Brasil em com- paração com os Estados Unidos, assemelhando-se ao de países do Leste Europeu.

De acordo com Lolio e Laurenti (24), que estudaram adultos maiores de 20 anos no Município de São Paulo, as taxas de mortalidade por DIC apresentaram tendência de aumento entre 1950 e 1976, seguida de declínio, especialmente no sexo feminino e nos mais jovens. De 1979 a 1989 observou-se um declínio desigual da mortalidade por DIC e DCBV em diversas capitais brasileiras (25). Entretanto, no nosso estudo, a capital do Rio de Janeiro apresentou tendência de estabilidade nas taxas brutas de mortalidade por DAC e declínio nas padronizadas, devido ao efeito da idade.

No presente trabalho, que se refere ao período de 1980 a 2006, o Estado do Rio de Janeiro e sua capital apresentaram taxas ajustadas e compensadas de mortalidade por DAC e DCBV mais altas do que os estados de São Paulo e Rio Grande do Sul e suas capitais. Por outro lado, a tendência de declínio nessas taxas foi mais acentuada no Estado do Rio de Janeiro e na sua capital.

O Estado do Rio de Janeiro apresentou as maiores taxas de mortalidade por DIC até o início da década de 1990. A partir de então, as taxas de mortalidade por DIC no Estado do Rio de Janeiro foram se aproximando daquelas dos outros dois estados. Nas capitais não houve um predomínio claro quanto à mortalidade por DIC, ainda que, nos últimos anos, as taxas do Município do Rio de Janeiro tenham sido as mais baixas. Portanto, é possível supor que o aumento das taxas de mortalidade por DIC tenha ocorrido durante a década de 1970, com estabilização na década seguinte e queda a partir da década de 1990.

Antes de considerar as possíveis explicações para esses achados, é importante refletir sobre a qualidade das informações sobre mortalidade, a qual pode ser avaliada pela análise da mortalidade por causas mal definidas. Destaca-se o aumento da mortalidade por essas causas no Estado e Município do Rio de Janeiro a partir de 1990, o que nos levou a calcular taxas de mortalidade compensadas, além de ajustadas por sexo e idade, para fazer as comparações entre os estados e os municípios. O aumento da mortalidade por causas mal definidas no Estado e no Município do Rio de Janeiro pode estar relacionado com uma portaria da Secretaria de Estado de Saúde, de 
FIGURA 3. Mortalidade por doenças cerebrovasculares compensada e ajustada nos estados (A) e capitais (B) do Rio de Janeiro, Rio Grande do Sul e São Paulo em adultos de 20 anos ou mais, 1980 a $2006^{a}$
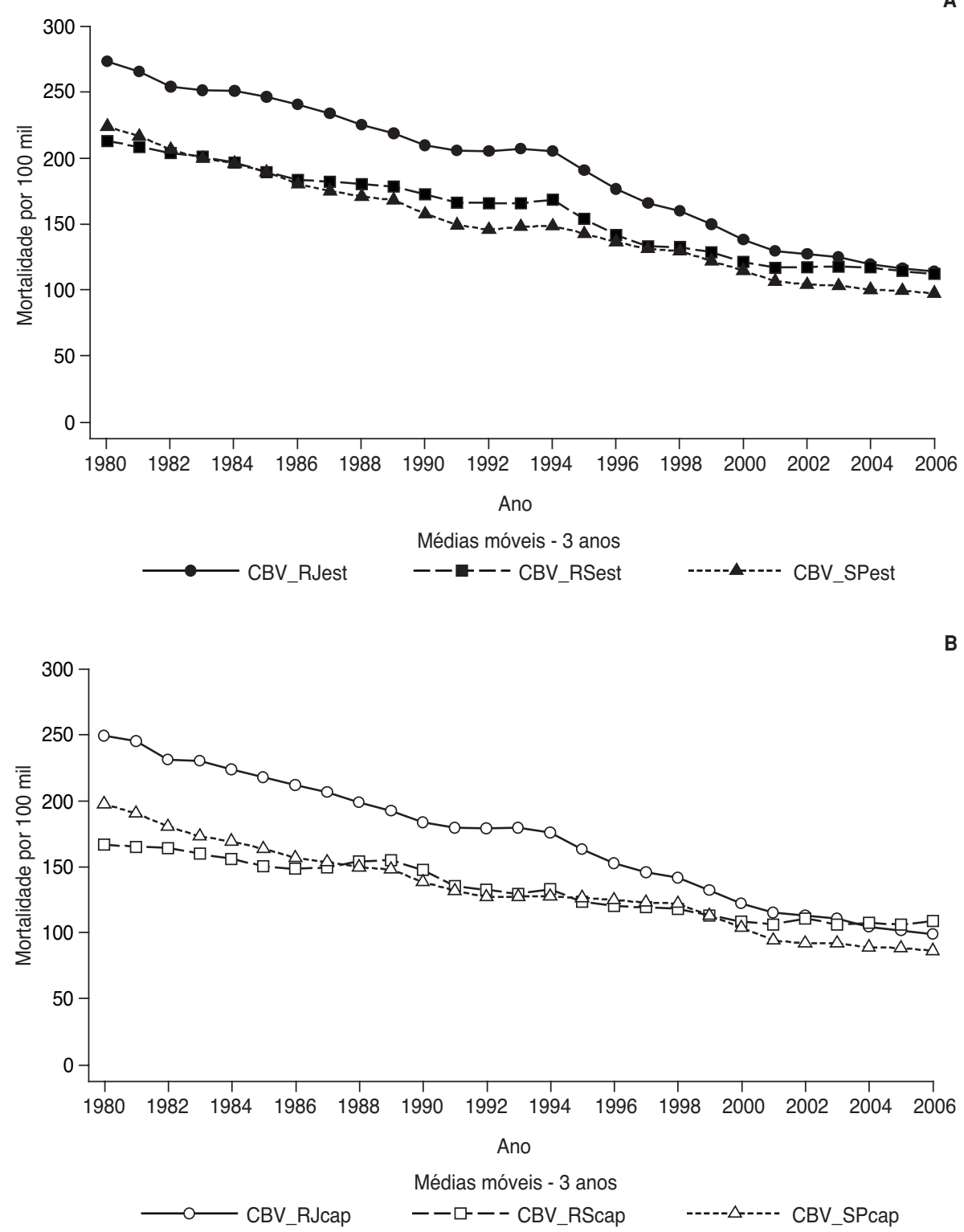

a RJcap = capital do Rio de Janeiro; RJest = Estado do Rio de Janeiro; RScap = capital do Rio Grande do Sul; RSest = Estado do Rio Grande do Sul; SPcap = capital de São Paulo; SPest = Estado de São Paulo.

29 de janeiro de 1990, que determinou: "esgotadas todas as tentativas de se determinar a causa básica da morte e não havendo suspeita de óbito por causa violenta (acidente, homicídio ou suicídio), deverá ser declarada na parte 1 do atestado médico causa indeterminada" (26).

É possível que a compensação dos óbitos por causas definidas com parte dos óbitos por mal definidas resulte em superestimação ou subestimação das taxas pelo maior controle dos fatores de risco clássicos, como observado no estudo MONICA (27). Os procedimentos de alta tecnologia (cirurgia de revascularização do miocárdio ou angioplastia) tampouco justificam as reduções de mortalidade observadas, uma vez que foram introduzidos nos últimos anos da década de 1960. Nos países em desenvolvimento, essas técnicas não são acessíveis à grande maioria da população; além disso, seus resultados na prática clínica não parecem tão efetivos (28). No Estado do Rio de Janeiro, a letalidade hospitalar por revascularização do miocárdio foi, em média, 7,5\% entre 1999 e 2003 (28). Nos países desenvolvidos, como por exemplo os Estados Unidos (29), a letalidade associada à revascularização em 2000 e 2001 variou de 2,4 a 3,5\%, de acordo com o número de cirurgias. Uma década antes, entre 1991 e 1993 (30), a letalidade pós-operatória imediata foi maior, variando de 3,6 a 5\%.

Podemos supor que a redução observada nas taxas de mortalidade cardiovascular poderia estar mais relacionada com a menor exposição a agentes infecciosos, devido às melhorias das condições de vida das populações. Essa sim, precedeu a queda da mortalidade cardiovascular.

O crescimento econômico ocorrido no Brasil entre 1930 e 1980 (31), apesar da concentração de renda, possibilitou a oferta de serviços de água e esgoto, com redução de doenças infecciosas e de processos inflamatórios decorrentes (32-35). Em países desenvolvidos, o declínio da mortalidade por DAC iniciouse cerca de 15 anos após o término da segunda guerra mundial, que sucedeu a grande depressão ocorrida no início da década de 1930 e a pandemia de influenza de $1918(36,37)$. No Brasil, esse mesmo declínio começou pouco mais de 40 anos após o início do período de crescimento econômico (31). A exposição a agentes infecciosos e outras condições insalubres nos primeiros anos de vida pode tornar os indivíduos mais suscetíveis ao desenvolvimento da aterotrombose vascular (32-35). A queda da mortalidade infantil precedeu a da mortalidade por DAC. É possível também que a redução da exposição às doenças infecciosas nas fases iniciais da vida esteja relacionada com o declínio observado na mortalidade cardiovascular dos adultos. Essas relações sinalizam a im- 
FIGURA 4. Mortalidade por doenças isquêmicas do coração compensada e ajustada nos estados (A) e capitais (B) do Rio de Janeiro, Rio Grande do Sul e São Paulo em adultos de 20 anos ou mais, 1980 a $2006^{a}$

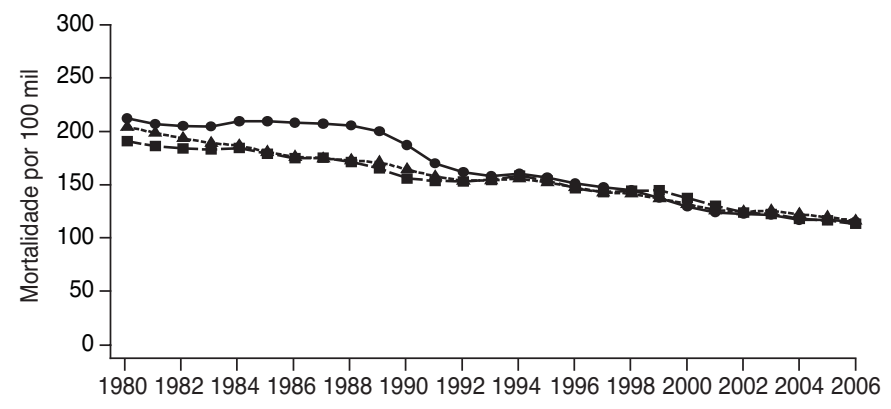

Ano

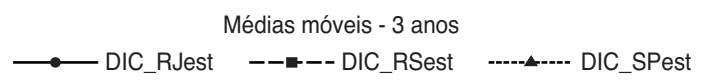

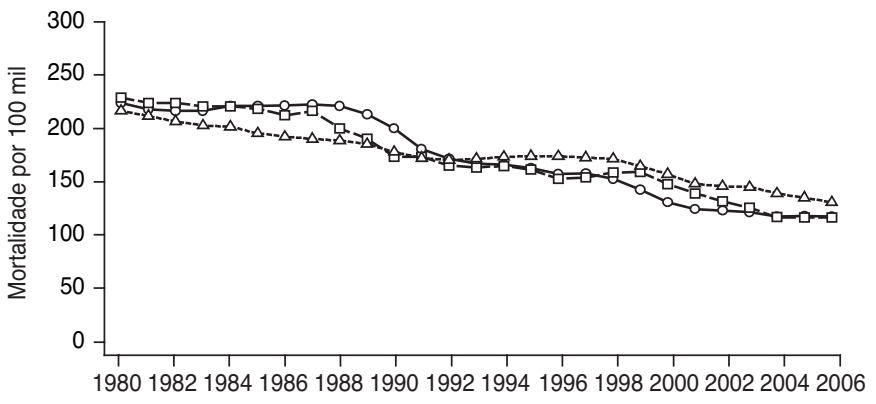

Ano

Médias móveis - 3 anos

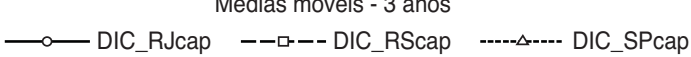

a RJcap = capital do Rio de Janeiro; RJest = Estado do Rio de Janeiro; RScap = capital do Rio Grande do Sul; RSest =D Estado do Rio Grande do Sul; SPcap = capital de São Paulo; SPest = Estado de São Paulo.

portância na melhoria de condições de vida das populações para reduzir a mortalidade cardiovascular (18).

Em síntese, os resultados aqui descritos sugerem que a queda da mortalidade ajustada por todas as causas observada nas últimas décadas se deveu principalmente ao declínio da mortalidade por DAC, precedida por queda na mortalidade por doenças infecciosas e pela me- lhoria das condições de vida da população. A queda da mortalidade por DAC se deveu em grande parte à redução da mortalidade por DCBV, notadamente no Rio de Janeiro.

\section{REFERÊNCIAS}

1. Prata PR. The epidemiologic transition in Brazil. Cad Saude Publica. 1992;8(2):168-75.

2. Yunes J, Ronchezel VSC. Evolução da mortalidade geral, infantil e proporcional no Brasil. Rev Saude Publica. 1974;8(supl):3-48.

3. Brasil, Ministério da Saúde, Secretaria Executiva. DATASUS. Informações de saúde. Morbidade e informações epidemiológicas. Disponível em: www2.datasus.gov.br/DATASUS/index. php?area $=0203$. Acessado em janeiro de 2008.

4. Godoy MF, Lucena JM, Miquelin AR, Paiva FF, Oliveira DLQ, Augustin Junior JL, et al. Mortalidade por doenças cardiovasculares e níveis socioeconômicos na população de São José do Rio Preto, Estado de São Paulo, Brasil. Arq Bras Cardiol. 2007;88(2):200-6.

5. Lotufo PA, Lolio CA. Tendência da mortalidade por doença isquêmica do coração no Estado de São Paulo: 1970 a 1989. Arq Bras Cardiol. 1993;61(3):149-53.

6. Lolio CA, Souza JMP, Laurenti R. Decline in cardiovascular disease mortality in the city of São Paulo, Brazil, 1970 to 1983 . Rev Saude Publica. 1986;20(6):454-64.

7. Passos LC, Lopes AA, Lessa I, Sanches A, Santos-Jesus R. Tendência da mortalidade por infarto do miocárdio (1981 a 1996) na cidade de Salvador, Brasil. Arq Bras Cardiol. 2000; 74(4):329-31.

8. Moraes SA, Rezende MHV, Freitas ICM. Tendência da mortalidade por doença isquêmica do coração no município de Goiânia-
Brasil na série histórica entre 1980 e 1994. Arq Bras Cardiol. 2000;74(6):493-7.

9. Governo do Estado do Rio Grande do Sul. Indicadores e dados básicos de saúde. Disponível em: www.saude.rs.gov.br/cronicos degenerativos/cronicos_degenerativos.php. Acessado em janeiro de 2009.

10. Kuulasmaa K, Tunstall-Pedoe H, Dobson A, Fortmann S, Sans S, Tolonen H, et al. Estimation of contribution of changes in classic risk factors to trends in coronary-event rates across the WHO MONICA Project populations. Lancet. 2000;355(9205):675-87.

11. Oliveira GMM, Klein CH, Silva NAS. Mortalidade por doenças cardiovasculares em três estados do Brasil de 1980 a 2002. Rev Panam Salud Publica. 2006;19(2):85-93.

12. Mansur AP, Favarato D, Souza MFM, Avakian $\mathrm{SD}$, Aldrighi JM, Cesar LAM, et al. Tendência do risco de morte por doenças circulatórias no Brasil de 1979 a 1996. Arq Bras Cardiol. 2001; 76(6):497-503.

13. Santo AH. Potencial epidemiológico da utilização das causas múltiplas de morte por meio de suas menções nas declarações de óbito, Brasil, 2003. Rev Panam Salud Publica. 2007; 22(3):178-86.

14. Organização Mundial de Saúde. Manual da Classificação Internacional de Doenças, Lesões e Causas de Óbitos, Nona Revisão, 1975. São Paulo: Centro da OMS para Classificação das Doenças em Português; 1978.
15. Organização Mundial de Saúde. Classificação estatística internacional de doenças e problemas relacionados à saúde: Classificação Internacional de Doenças. Décima Revisão. Vol. 1. São Paulo: EDUSP; 1995.

16. Vermelho LL, Costa AJL, Kale PL. Indicadores de saúde. Em: Medronho RA. Epidemiologia. $2^{\mathrm{a}}$ ed. São Paulo; Editora Atheneu; 2008. Pp. 33-57.

17. Pagano M, Gauvreau K. Princípios de bioestatística. São Paulo, Pioneira Thomson Learning; 2004.

18. Soares GP, Brum JD, Oliveira GMM, Klein $\mathrm{CH}$, Nelson Albuquerque de Souza e Silva. Mortalidade por doenças isquêmicas do coração, cerebrovasculares e causas mal definidas nas regiões do estado do Rio de Janeiro, 1980-2007. Rev SOCERJ. 2009;22(3):142-50.

19. Morettin PA, Toloi CMC. Análise de séries temporais. São Paulo, Edgard Blücher; 2006.

20. Lotufo PA. Por que não vivemos uma epidemia de doenças crônicas: o exemplo das doenças cardiovasculares? Cienc Saude Coletiva. 2004;9(4):844-7.

21. Laurenti R, Gotlieb SL, Souza JMP, Fonseca LA, Jorge MH. Características da mortalidade por doença isquêmica do coração em adultos de 15 a 74 anos no município de São Paulo. Arq Bras Cardiol. 1981;36(2):85-9.

22. Laurenti R. Epidemiologia das doenças cardiovasculares no Brasil. Arq Bras Cardiol. 1982; 38(4):243-8. 
23. Chor D, Fonseca MJ, Andrade CR. Doenças cardiovasculares. Comentários sobre a mortalidade precoce no Brasil. Arq Bras Cardiol. 1995;64(1);15-9.

24. Lolio CA, Laurenti R. Mortalidade por doença isquêmica do coração no município de São Paulo. Evolução de 1950 a 1981 e mudanças recentes na tendência. Arq Bras Cardiol. 1986; 46(3):153-6.

25. Lolio CA, Lotufo PA, Lira AC, Zanetta DM, Massad E. Tendência da mortalidade por doença isquêmica do coração nas capitais de regiões metropolitanas do Brasil, 1979-89. Arq Bras Cardiol. 1995;64(3):213-6.

26. Governo do Estado do Rio de Janeiro. Portaria $\mathrm{n}^{\circ} 550$ de 23 de janeiro de 1990. Em: Brasil. Diário Oficial da União de 29 de janeiro de 1990. Brasília: Imprensa Nacional; 1990.

27. Tunstall-Pedoe H, ed, Kuulasmaa K, Tolonen $\mathrm{H}$, Davidson M, Mendis S with 64 other contributors for The WHO MONICA Project. MONICA Monograph and Multimedia Sourcebook. Genebra: WHO; 2003. Disponível em: www.ktl.fi/monica/public/monograph.html. Acessado em setembro de 2009.
28. Godoy $\mathrm{PH}$, Klein $\mathrm{CH}$, Souza e Silva NA, Oliveira GMM, Fonseca TMP. Letalidade na cirurgia de revascularização do miocárdio no Estado do Rio de Janeiro-SIH/SUS-no período de 1999-2003. Rev SOCERJ. 2005;18(1): 23-9.

29. Peterson ED, Coombs LP, DeLong ER, Haan CK, Ferguson TB. Procedural volume as a marker of quality for CABG surgery. JAMA. 2004;291(2):195-201.

30. Clark RE. Outcome as a function of annual coronary artery bypass graft volume. The Ad Hoc Committee on Cardiac Surgery Credentialing of The Society of Thoracic Surgeons. Ann Thorac Surg. 1996;61(1):21-6.

31. Lesbaupin I, Mineiro A. O desmonte da nação em dados. Petrópolis: Vozes; 2002.

32. Finch CE, Crimmins EM. Inflammatory exposure and historical changes in human lifespans. Science. 2004;305(5691):1736-9.

33. Bloom G. Equity in health in unequal societies: towards health equities during rapid social changes. IDS Working Paper 112; 2000.

34. World Development Report 2000/2001-Attacking poverty. Oxford: Oxford University Press/World Bank; 2000.
35. United Nations Economic and Social Council. Report of the Secretary General: health and sustainable development (E/CN.17/2001/ PC/6). Nova Iorque: CSD; 2001.

36. Azambuja MI. Connections: can the 20th century coronary heart disease epidemic reveal something about the 1918 influenza lethality? Braz J Med Biol Res. 2008;41(1):1-4.

37. Azambuja MI, Achutti A, Levins R. The inflammation paradigm: Towards a consensus to explain coronary heart disease mortality in the 20th century. CVD prevention and control. 2008;3(2):69-76.

Manuscrito recebido em 28 de outubro de 2009. Aceito em versão revisada em 10 de junho de 2010.
ABSTRACT

\section{All-cause and cardiovascular diseases mortality in three Brazilian states, 1980 to 2006}

Objective. To evaluate mortality from all causes, diseases of the circulatory system (DCS), ischemic heart disease (IHD), and cerebrovascular diseases (CVD) from 1980 to 2006 in Rio de Janeiro, Rio Grande do Sul, São Paulo, and their capitals, taking into consideration the impact of deaths due to ill-defined causes.

Methods. Population and mortality data were obtained from the Unified Health System's Data Bank (DATASUS). Mortality from the diseases of interest and from illdefined causes was adjusted by the direct method for adults older than 20 years of age. Since the mortality rates from ill-defined causes increased markedly after 1990, proportional mortality rates from ill-defined causes were calculated. Linear regression models were used for analysis of trends.

Results. A relevant decline in all-cause mortality was observed in the three states and capitals. Rio de Janeiro and its capital had the highest rates of all-cause mortality. DCS mortality declined more than all-cause mortality. Proportional mortality from illdefined causes in Rio de Janeiro and its capital was higher than in all other states and capitals starting in 1990. CVD mortality fell in the study period, especially in Rio de Janeiro and its capital. The state of Rio de Janeiro also had the highest IHD mortality rates until 1993. Among the capitals, São Paulo presented the highest IHD mortality rates starting in 1992.

Conclusions. The decline in all-cause mortality resulted mainly from the decline in DCS mortality. In turn, the decline in DCS mortality was partly due to the reduction in CVD mortality, especially in the state of Rio de Janeiro.

Key words Mortality; cause of death; cardiovascular diseases; myocardial ischemia; cerebrovascular disorders; epidemiology; Brazil. 